\title{
LE VERTIGE DU VIDE. LA FICTION MINIMALISTE DE JEAN ECHENOZ
}

\author{
Anna Maziarczyk
}

\begin{abstract}
Associated with minimalist art due to its stylistic circumspection and impassivity, minimalist writing simultaneously returns to and modifies traditional storytelling techniques. Inspired by everyday, banal reality, Jean Echenoz creates seductive fictions, which mix classic novelistic genres and engage in an intertextual game with the tradition via numerous allusions and quotations. His literary aesthetics relies on the contrast between the baroque plot, multiplying improbable adventures, and concise narration, exploiting blank space, discontinuity and arbitrariness, which destabilise logical continuity and undermine textual equilibrium. Subverting the standard novelistic rules, Echenoz creates modern fiction, which is paradoxically minimalist and vertiginous.
\end{abstract}

Keywords: Jean Echenoz; minimalist writing; void; blank space; vertigo.

Résumé : Associée à l'art minimaliste pour cause de la sobriété stylistique et de l'impassibilité, l'écriture minimaliste favorise la fiction, tout en la réinventant en même temps. S'inspirant de la réalité quotidienne et banale, Jean Echenoz écrit des romans séduisants qui mixent des sous-genres romanesques classiques et entament un jeu intertextuel avec la tradition à travers multiples allusions et citations. Son esthétique littéraire repose sur un contraste entre l'intrigue baroque qui multiplie les situations rocambolesques et la narration concise exploitant le blanc, le discontinu et l'arbitraire qui déstabilisent la continuité logique et entament l'équilibre textuel. Subvertissant les règles romanesques en vigueur, Echenoz crée une fiction tout à fait moderne, paradoxalement minimaliste et vertigineuse.

Mots clés : Jean Echenoz ; écriture minimaliste ; vide ; blanc ; vertige.

L'écriture des jeunes auteurs publiés par les éditions de Minuit à partir des années 1980 s'appuie sur un paradoxe qui assure son caractère original et (post)moderne. Associée à l'art minimaliste pour cause de la sobriété stylistique et de l'impassibilité, elle recherche un style neutre, dépourvu d'ornements inutiles, permettant de saisir la réalité de plus près, dans son état brut. Or, même si « l'écriture minimaliste relève davantage d'une éthique de la forme » (Blanckeman 2002 : 66), elle s'inscrit dans la tendance caractéristique de la littérature de cette période qu' on nomme « le retour au récit ». Favorisant la fiction, elle la réinvente en même temps et les stratégies narratives subissent un changement notoire pour satisfaire ce « simple désir de raconter » (Viart 1993 : 153).

L'esthétique littéraire de Jean Echenoz, considéré comme le chef de file de l'école minimaliste, repose sur un contraste tout à fait particulier entre l'intrigue aventureuse, voire même quasi baroque, et la narration très concise qui trouve son mode préféré d'expression dans le blanc, le discontinu et l'arbitraire. Appellant l'investissement du lecteur 
dans la construction du sens, le texte le manipule en même temps en multipliant les situations rocambolesques et étranges qui se suivent à un rythme fulgurant. Contribuant au vertige textuel, le vide constitue un élément inhérent du jeu littéraire qui se déroule à plusieurs niveaux du texte, tout en assurant les émotions de lecture engendrées par les mystères autour desquels gravite l'action et un plaisir intellectuel du déchiffrement des énigmes.

Chez Echenoz, la passion pour la fiction se manifeste notamment par un goût du romanesque dans le sens le plus classique de ce terme, romanesque qui est cependant suberti par un ordre narratif atypique pour le genre en question. Au premier abord, on peut considérer ses textes comme des histoires futiles d'une grande banalité : des personnages plutôt plats privés de motivations psychologiques sont impliqués dans des actions tantôt insignifiantes, tantôt tout à fait abracadabrantes. Organisée autour d'un mystère ou d'une énigme, l'intrigue se déroule à travers des péripéties invraisemblables qui font bifurquer la banalité initiale vers le fantastique voire l'absurde. Exploitant diverses formes du récit, surtout le roman policier, le roman d'espionnage, le récit d'aventures ou bien la science-fiction ${ }^{1}$, Echenoz les combine dans diverses configurations et procède à des variations formelles dignes d'un prestigitateur de genres.

L'Equipée malaise constitue, à ce titre, un exemple des plus significatifs (Echenoz 1986). Organisateur d'une révolte contre les propriétaires de la plantation de Malacca en Malaisie, Pons se déplace à Paris dans le but de se procurer les armes nécessaires. Les trafiquants qu'il contacte à ce sujet dont l'un est, par ailleurs, son neveu, sont eux-mêmes poursuivis par des criminels pour des affaires louches. L'affaire conclue, suit le voyage de retour en bateau, d'autant plus dramatique que l'équipe doit faire face tout d'abord aux périples de nature, tels une violente tempête, pour ensuite passer à la confrontation personnelle, le malaise évoqué dans le titre se référant également aux relations humaines entre les passagers. La traversée de l'océan devient ainsi un vrai «voyage au bout de la nuit (avec les tropiques comme destinée de l'enfer) » (Houppermans 2008 : 41) et à l'intrigue policière du début succède le roman d'aventures fusionnant avec le récit d'apprentissage. Cette hétérogénéité générique est complétée par le roman d'amour des plus romantiques et incroyables : la retrospection par lequelle débute le roman évoque une vieille et touchante histoire d'un triangle dans lequel ont été impliqués les principaux protagonistes Pons et Charles, amoureux dans leur jeuneusse de la même fille, une certaine Nicole Ficher. L'histoire procédant par répétitions, quelques trente années plus tard la jeune génération revit les émotions analogues quand les copains Paul et Bob s'éprennent tous les deux de Justine, par ailleurs la fille de ladite Nicole. Les épisodes aventureux tels des fuites et des poursuites, des enlèvements et des séquestrations, des rencontres biscornues et des disparitions étranges s'entrecroisent avec des péripéties sentimentales classiques comme des coups de foudre et des élans de cœur, des promesses de fidélité et des ruptures, des départs et des divorces. Avançant ainsi à un rythme impétueux, l'intrigue fait suspendre l'haleine et suscite les émotions tout comme le font les grands romans d'aventures.

En transgressant les règles traditionnelles qui détérminent les genres littéraires, Echenoz entrave le lisibilité du texte et déroute le lecteur, en le privant d'indications jugées

\footnotetext{
1 Echenoz reconnaît la création de Jean-Pierre Manchette, principal représentant du polar français, comme une de ses principales inspirations littéraires. Cf. «Jean Echenoz, romancier de l'errance », Le Magazine littéraire, $\mathrm{n}^{\circ}$ 453, 2006.
} 
par Eco comme nécessaires pour la perception du texte (Eco : 1985). Flirtant avec la tradition du roman, Echenoz révèle par là-même sa passion de la narration et le foisonnement de l'imaginaire : il reprend les genres classiques pour les recomposer, élargir et subvertir dans un récit vertigineux par sa dynamique et tout à fait contemporain dans sa forme. Le retour au récit $n$ 'implique pas forcément la reprise de la narration naïve, tout au contraire : avec les composantes classiques du roman, telles l'histoire et les personnages, le récit est structuré selon une nouvelle esthétique qui valorise surtout l'absence et le fragmentaire. Plusieurs réflexions contemporaines constatent cette prédilection : ainsi Pascal Quignard affirme que « on peut soutenir que de nos jours la cheville ou le poncif, $c^{\prime}$ est le blanc. La règle paraît être un texte comme haillonneux. Du moins dans l'art moderne l'effet de discontinu s'est substitué à l'effet de liaison » (Quignard 1986 : 20).

S'inscrivant dans cette tendance caractéristique de la création artistique contemporaine, l'écriture de Jean Echenoz repose sur le blanc - aussi bien le blanc formel que syntaxique et narratif. Ses romans, en général brefs en ce qui concerne les dimensions, revêtent une structure morcelée soulignée encore davantage par la disposition du texte sur la page. Découpés par de nombreux alinéas qui font ressortir la présence des surfaces vides, les chapitres semblent se suivre quelque peu par hasard, sans transitions qui évoqueraient des relations causales entre eux, donnant ainsi l'impression d'un rassemblement gratuit d'événements. Privé de cohésion interne, le récit accorde une importance considérable à l'espace libre, potentiel, ouvert à des intreprétations. Cette structure à trous est surtout visible dans L'Equipée malaise dont l'organisation suit le travail de la mémoire de Paul, centré sur la reconstuction des souvenirs qui manquent :

[...] ses souvenirs de cette lettre n'étaient que miscellanées en vrac où l'affectif se bousculait sans méthode au météorologie, l'éthique ou géopolitique, l'autobiographique à l'astral comme dans un sac de linge. Avec, lui avait-il semblé, beaucoup de citations de mémoire, de points d'exclamation et de suspension, de parenthèses béantes et de questions laissées en friche. (Echenoz 1986 : 103).

Tout comme la mémoire de Paul, l'intrigue du roman bifurque dans des directions divergentes, entrecoupée d'histoires apparemment insignifiantes et de digressions falotes. Se ramifiant en une sorte de rhizome, le texte revêt quelque structure indeterminée et inachevée qui s'esquive aux essais de totalisation, d'autant plus qu'il est dépourvu de dénouement classique.

De manière paradoxale, le manque apparaît également comme un élément significatif de l'intrigue, thématisé dans les textes d'Echenoz à plusieurs reprises. L'histoire même de certains romans tourne autour de ce motif, habilement exploité tant dans le roman policier (le manque d'armes nécessaires dans L'Equipée malaise ou la disparition de Gloire Abgrall, étoile éphémère du «showibz » dans Les grandes blondes) que dans le roman d'amour (l'absence de la femme aimée dans Nous trois). Dans ce dernier cas, les réflexions sur le vide causé par le temps qui passe et sur la solitude de l'homme produisent l'effet d'autant plus émouvant qu'elles sont exprimées sans délayage émotionnel, avec une concision tout à fait minimaliste, en constituant également un principe essentiel du roman entier. Le vide rythme tous les romans d'Echenoz sous forme du leitmotif de chute dont l'apparition cadencée structure la narration. Rappelons ici, à titre d'exemple, nombreux accidents de ce type qui arrivent surtout à des détectives chargés de missions délicates, tels Jean-Claude Kastner ou Gilbert Flon qui basculent d'un rocher dans un abîme. L'effet dramatique lié au vertige est d'autant plus violent que les scènes sont re- 
flétées dans des rêves à caractère prophétique (la peur du vertige de Kastner s'exprime à travers des cauchemars qui le tourmentent régulièrement), multipliant ainsi le danger et l'angoisse devant l'inévitable.

L'idée du manque semble par ailleurs hanter de manière quasi obsessionnelle le narrateur même puisqu'il note scrupuleusement les évènements qui ne se sont pas produits ou les mots qui n'ont pas été prononcés. Il constate, par exemple, l'absence d'objets, d'émotions ou de bruits : «nul mouvement nulle part [...], nul bruit non plus » (Echenoz 1986 : 173). La dialectique de la présence et de l'absence devient ainsi l'axe principal de l'écriture échenozienne tant au niveau de l'histoire, où elle apparaît comme un motif privilégié, qu'à celui de la structure textuelle qui joue sur l'espace imprimé et le blanc, pour enfin influer sur les techniques narratives.

Sans se faire repérer par des marques formelles, les blancs narratifs ponctuent régulièrement les textes analysés, en les sapant de l'intérieur et en détruisant encore davantage leur cohésion fragile. Les romans policiers ou d'espionnage, conformément aux règles de ces genres, font l'économie de certaines données afin de produire l'effet d'énigmaticité et, jouant sur le suspens, ils invitent le lecteur à effectuer le travail du déchiffrement du texte. Selon Barthes, la dissimulation est une stratégie principale dans ce type de récit puisqu'il

[...] doit disposer dans le flux du discours des retards (chicanes, arrêts, dévoiements); sa structure est essentielement réactive, car il oppose à l'avancée inéluctable du langage un jeu échelonné d'arrêts : c'est, entre la question et la réponse, tout un espace dilatoire, dont l'emblème pourrait être la "réticence », cette figure rhétorique qui interrompt la phrase, la suspend et la dévie [...]. (Barthes $1970: 82$ ).

Le narrateur ménage donc son savoir de sorte à ne pas trahir les informations qui feraient progresser l'intrigue trop vite et en révéleraient l'enjeu. Le récit est organisé autour de la parataxe, procédé qui " manifeste des séries de trous, d'absences, de silences noués où se scellent les non-dits et les implicites » (Scepi 1990 : 1025). Dissimulant certains faits importants pour le déroulement de l'action, la parataxe fait également endommager les rapports logiques entre les événements. Par conséquent, le récit perd sa cohérence et, tout en restant toujours plutôt linéaire, il se dissloque en bribes dépourvues d'une motivation causale. Cette notion de fragmentation devient par ailleurs thématisée au niveau du récit même à travers le motif de la dissection des corps qui revient souvent dans les textes d'Echenoz. Rappelons ici deux lieux privilégiés du Lac qui sont, à ce titre, significatifs : il s'agit de l'abattoir et du cimetière, les espaces où ce processus de la désintégration corporelle est poussé à l'extrême.

Désintégrée elle aussi par le manque d'enchaînements logiques, l'intrigue devient une suite incohérente d'images qui semblent être régies par des lois arbitraires. Avec sa structure décousue, elle se présente comme une succession chaotique de hasards et coïncidences, ce que Fink appelle le « jeu du monde » (Fink 1966). Or, comme le constate Fieke Schoots «le récit minimaliste est parcouru par plusieurs lignes qui ne peuvent pas se résumer en une seule. [...] Au lieu d'être dissociées, elles entrent en connexion [...]. Les transitions d'une ligne à une autre ont lieu sans que le cours de l'histoire soit interrompu » (Schoots 1997 : 137). Lézardée par des failles qui en compromettent l'intégrité, la surface du texte forme un faisceau de fils qui s'entrecroisent tout en se ramifiant en même temps. Les blancs et les non-dits endommagent l'équilibre textuel de manière astucieuse : à peine perceptibles, ils sont rapidement anéantis par le flux de nouvelles 
données qui apparaissent au moment où la narration bifurque dans une autre direction. Se succédant à un rythme frénétique, les arrêts et les envols abrupts de l'intrigue engloutissent le lecteur dans un vertige textuel où les points de répères deviennent insaisissables. Les grandes blondes constitue, à ce titre, un des meilleurs exemples : la mission qui consiste à retrouver Gloire Abgrall, médiocre vedette qui a renoncé à sa carrière artististique, devient un vrai ballet de fuites et de poursuites, entrecoupé par des disparitions mystérieuses et des incidents aberrants, des meurtres brutaux et des scènes tout à fait fantastiques. A chaque moment où les détectives sont sur le point de découvrir le domicile temporaire de la vamp blonde, elle s'échappe pour se retrouver, déguisée, à l'autre bout du monde, accompagnée toujours de son ami tant bizarre que malin - nain d'une trentaine de centimètres nommé Béliard, un véritable diablotin qui apparaît dans le texte à des intervalles irréguliers. Ponctuée d'événements tant absurdes que comiques, comme par exemple l'histoire du trafic illégal des drogues dans lequel Gloire est impliquée malgré elle ou bien le miracle tout à fait abracadabrant qui fait sauver la vie à un agent précipité d'un rocher, l'histoire se termine par un happy end - un amour naissant entre la fugitive et son pourchasseur, réalisation symbolique du désir que suscite la grande blonde, avatar de la féminité sensuelle mais inaccessible.

Outre ces rebondissements et détournements rocambolesques, le récit échenozien se développe par une série d'écarts, à savoir des digressions, des commentaires et des épisodes sans rapport évident avec les événements qui se déroulent. Dans Le Méridien de Greenwich, l'abondance de personnages secondaires et la profusion de péripéties secondaires font constamment intrusion à l'intrigue principale, rien donc d'étonnant que la recherche d'une machine secrète se termine par un échec d'autant plus spectaculaire que le fameux instrument s'avère n'être qu'un leurre, "pure apparence, contenant vide et fortel, efficace comme peut l'être un accessoire de théâtre » (Echenoz 1979 : 221). Plusieurs épisodes de L'Equipée malaise semblent être sans intérêt pour l'histoire principale, comme c'est le cas de la description de la pointure des chaussures de Jouve ou de l'énumération du contenu de la boîte à lettres (Echenoz 1986 : 12, 55). Le récit consistue ainsi un prétexte à toutes les divagations qui, d'une part, prouvent un réel plaisir que le narrateur éprouve du simple fait de raconter, d'autre part cependant brouillent la lisibilité de l'histoire. Comme le constate Sjef Houppermans, « le tout fonctionne donc comme un processus de rapprochement et de distanciation, thérapie de choc et laboratoire holographique, équivalent littéraire dans un sens des sauts à l'élastique. A trop se rapprocher du fascinant l'objet du désir celui-ci perd ses contours, sa délimitation, et donc sa réalité [...] » (Houppermans 1999 : 22). En même temps, l'excès de détails dissimule l'absence d'informations nécessaires car les bribes fournies par le narrateur ne sont pas suffisantes pour combler les vides qui apparaissent dans la trame de la narration. « La prolixité narrative dynamise le récit et provoque une lecture orientée tout en pédalant dans le vide » (Jerusalem 2005 : 20).

Les errances narratives se manifestent également sous forme de l'écriture oblique (Hamon 1996) qui fait toute la saveur du style d'Echenoz. Maniant un langage simple sans se soucier trop des règles de la syntaxe et de la ponctuation, il mène son récit de manière quelque peu désinvolte, prenant toutes ses distances par rapport à l'histoire qu'il raconte à travers des phrases parfois grammaticalement incorrectes, inachevées ou elliptiques. Les défaillances et les blancs dans la narration sont adroitement masqués par l'érudition des références intertextuelles et des citations dont les romans sont parsemés. Conformé- 
ment à l'esthétique contemporaine qui est celle de la disparition (Virilio 1989; Barthes 1984), le narrateur s'abstient de s'exprimer et préfère céder la parole aux autres artistes, sans craindre le risque d'anéantissement auquel il expose ainsi son œuvre (Compagnon 1990 : 167). Bien au contraire : les références intertextuelles creusent le texte et le mettent en contact avec toute la culture antérieure, en dévoilant ainsi, de manière valorisante ou critique, des influences subies et des sources d'inspiration. Dans les romans d'Echenoz, on remarque l'ominiprésence surtout de la culture populaire, avec une prédilection évidente pour le cinéma. Ponctués de renvois aux films américains des années 50, Le Lac ou Les grandes blondes construisent ainsi un monde d'inspiration cinématographique où les titres des films, les noms des acteurs et les motifs cités construisent tout un réseau d'analogies. Créant «l'effet de remake qui hante le récit » (Houppermans 1999: 27), ces références s'agencent en un kaléidoscope de motifs récurrents qui ponctuent le récit, les images évoquées constituant une sorte de reflets de l'histoire racontée. Elles sont en même temps les figures fondamentales pour la poétique d'Echenoz à travers lesquelles, tout en flirtant avec la tradition, il déconstruit la narration classique et la reconstruit à sa propre manière. « Empêchant le regard à plat, l'enfermement dans le seul enchaînement des causalités » (Lebrun 1992 : 120), le jeu citationnel contribue à la discontinuité narrative puisqu'il fait superposer diverses instances narratives, tout en entraînant une multiplication de représentations à l'intérieur du récit. La confusion de niveaux diégétiques qui en est la conséquence fait brouiller encore davantage les points de repères. Par détournements, l'intrigue aboutit ainsi vers la fin qui ne constitue pas de dénouement au sens classique du terme et ne conclut point l'histoire racontée. $S^{\prime}$ il y a une résolution d'énigmes, c'est plutôt par un pur effet de hasard que grâce à une interprétation logique des faits ou bien une révélation finale du narrateur. A travers la réécriture, le texte s'inscrit dans un cercle vicieux de renvois infinis qui remodèlent constamment le sens, tout en laissant persister des zones d'ombres qui s'esquivent à l'explication.

Renouant avec la tradition du genre romanesque, Echenoz en remanie les enjeux et contribue au renouvellement de la fiction. $S^{\prime}$ inspirant de la réalité quotidienne et banale, il crée des romans particulièrement séduisants qui mixent des sous-genres romanesques classiques et entament un jeu intertextuel avec la tradition à travers des allusions, des citations et toute sorte de références parfois très subtiles qui ponctuent le récit. Les travestissements et les parodies à visée ludique poussent à l'extrême la dynamique de l'intrigue, elle-même impétueuse sinon baroque à cause des situations rocambolesques qui se succèdent à un rythme fulgurant. Ce vertige textuel est accentué encore davantage par la narration : appelée minimaliste non sans raison, elle trouve son mode préféré d'expression dans le blanc, le discontinu et l'arbitraire qui déstabilisent la continuité logique et entament l'équilibre textuel. Préférant l'ambiguïté à la démarche ostensiblement explicative et l'écriture désinvolte par son côté ironique à l'érudition moralisante, Echenoz subvertit les règles romanesques en vigueur et crée une fiction tout à fait moderne, paradoxalement minimaliste et vertigineuse.

\section{Bibliographie}

BARTHES, Roland (1970), S/Z, Paris : Seuil.

BARTHES, Roland (1984), Essais critiques IV. Le bruissement de la langue, Paris, Seuil.

BlancKeman, Bruno (2002), Les fictions singulières. Etude sur le roman français contemporain, Paris : Prétexte éditeur. 
Compagnon, Antoine (1990), Les cinq paradoxes de la modernité, Paris : Seuil.

ECHENOZ, Jean (1979), Le Méridien de Greenwich, Paris : Minuit.

ECHENOZ, Jean (1986), L'Equipée malaise, Paris : Minuit.

ECHENOZ, Jean (1992), Nous trois, Paris : Minuit.

ECHENOZ, Jean (1995), Les grandes blondes, Paris : Minuit.

Eco, Umberto, (1985), Lector in fabula, Paris : Grasset.

FINK, Eugène (1966), Le jeu comme symbole du monde, Paris : Minuit.

Hamon, Philippe (1996), L'ironie littéraire. Essai sur les formes de l'écriture oblique, Paris : Hachette.

Houppermans, Sjef (1999), «Les grandes blondes parmi les noirs - Jean Echenoz », in : BAETENS, Jan - Viart, Dominique (éds.), Ecritures contemporaines 2. Etats du roman contemporain, Paris - Caen, Lettres Modernes Mirand, 19-39.

Houppermans, Sjef (2008), Jean Echenoz, Paris : Bordas / SEJER.

«Jean Echenoz, romancier de l'errance », Le Magazine littéraire, n 453, 2006.

Jerusalem, Christine (2005), Jean Echenoz, Géographies du vide, Saint-Etienne : Publications de l'université de Saint-Etienne.

Lebrun, Jean-Claude (1992), Jean Echenoz, Paris : Éditions du Rocher.

Quignard, Pascal (1986), Une gêne technique à l'égard des fragments, Montpellier : Fata Morgana.

ScEPI, Henri (1990), «Patrick Deville: Pour une esthétique du dérèglement », Critique, vol. 46, n 523, pp. 1020-1041.

Schoots, Fieke (1997), Passer en douce à la douane. L'écriture minimaliste de Minuit, Amsterdam - Atlanta : Rodopi.

VIART, Dominique (1993), «Le récit postmoderne », in : VIART, Dominique - BAERT, Franck (éds.), La littérature française contemporaine, questions et perspectives, Louvain : Presses universitaires de Louvain,153-165.

VirILIO, Paul (1989), Esthétique de la disparition, Paris : Éditions Galilée.

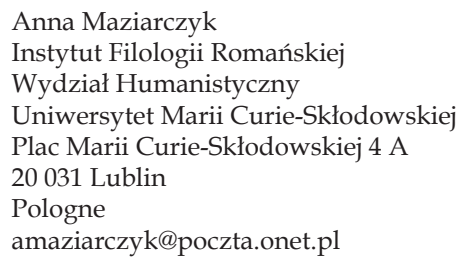

